\title{
A NECESSARY AND SUFFICIENT CONDITION FOR THE NONTRIVIAL LIMIT OF THE DERIVATIVE MARTINGALE IN A BRANCHING RANDOM WALK
}

\author{
XINXIN CHEN, ${ }^{*}$ Université Paris VI
}

\begin{abstract}
We consider a branching random walk. Biggins and Kyprianou (2004) proved that, in the boundary case, the associated derivative martingale converges almost surely to a finite nonnegative limit, whose law serves as a fixed point of a smoothing transformation (Mandelbrot's cascade). In this paper, we give a necessary and sufficient condition for the nontriviality of the limit in this boundary case.
\end{abstract}

Keywords: Branching random walk; derivative martingale; Mandelbrot's cascade; random walk conditioned to stay positive

2010 Mathematics Subject Classification: Primary 60J80

Secondary 60G42

\section{Introduction}

We consider a discrete-time branching random walk (BRW) on the real line, which can be described in the following way. An initial ancestor, called the root and denoted by $\varnothing$, is created at the origin. It gives birth to some children which form the first generation and whose positions are given by a point process $\mathcal{L}$ on $\mathbb{R}$. For any integer $n \geq 1$, each individual in the $n$th generation gives birth independently of all others to its own children in the $(n+1)$ th generation, and the displacements of its children from this individual's position are given by an independent copy of $\mathcal{L}$. The system continues if there is no extinction. We thus obtain a genealogical tree, denoted by $\mathbb{T}$. For each vertex (individual) $u \in \mathbb{T}$, we denote its generation by $|u|$ and its position by $V(u)$. In particular, $V(\varnothing)=0$ and $(V(u) ;|u|=1)=\mathcal{L}$.

Note that the point process $\mathcal{L}$ plays an important role in the BRW as the offspring distribution in a Galton-Watson process. We introduce the Laplace-Stieltjes transform of $\mathcal{L}$ as follows:

$$
\Phi(t):=\mathbb{E}\left[\int_{\mathbb{R}} \mathrm{e}^{-t x} \mathcal{L}(\mathrm{d} x)\right]=\mathbb{E}\left[\sum_{|u|=1} \mathrm{e}^{-t V(u)}\right] \quad \text { for all } t \in \mathbb{R} .
$$

Let $\Psi(t):=\log \Phi(t)$. In this paper we always assume that $\Psi(0)>0$ so $\mathbb{E}\left[\sum_{|u|=1} 1\right]>1$. This yields that, with strictly positive probability, the system survives. Let $q$ be the probability of extinction. Clearly, $q<1$.

Let $\left(\mathcal{F}_{n} ; n \geq 0\right)$ be the natural filtration of this BRW, i.e. let $\mathscr{F}_{n}:=\sigma\{(u, V(u)) ;|u| \leq n\}$. We introduce the additive martingale, for any $t \in \mathbb{R}$,

$$
W_{n}(t):=\sum_{|u|=n} \mathrm{e}^{-t V(u)-n \Psi(t)} .
$$

Received 4 March 2014; revision received 15 August 2014.

* Postal address: Laboratoire de Probabilités et Modèles Aléatoires, Université Paris VI, 4 Place Jussieu, 75005 Paris, France. Email address: luciechen1988@gmail.com 
It is a nonnegative martingale with respect to $\left(\mathcal{F}_{n} ; n \geq 0\right)$, which converges almost surely (a.s.) to a finite nonnegative limit. Biggins [3] established a necessary and sufficient condition for the mean convergence of $W_{n}(t)$. A simpler proof based on a change of measures was given later by [14].

More generally, Biggins and Kyprianou [6] studied the martingales produced by the socalled mean-harmonic functions. Given suitable conditions on the offspring distribution $\mathcal{L}$ of the BRW, like the $X \log X$ condition of the Kesten-Stigum theorem, Biggins and Kyprianou provided a general treatment to obtain the mean convergence of these martingales. In this paper, following their ideas, we work on one special example and provide a Kesten-Stigumlike theorem.

Throughout this paper, we consider the boundary case (in the sense of [7]), where $\Psi(1)=$ $\Psi^{\prime}(1)=0$, i.e.

$$
\mathbb{E}\left[\sum_{|u|=1} \mathrm{e}^{-V(u)}\right]=1, \quad \mathbb{E}\left[\sum_{|u|=1} V(u) \mathrm{e}^{-V(u)}\right]=0 .
$$

In addition, we assume that

$$
\sigma^{2}:=\mathbb{E}\left[\sum_{|u|=1} V(u)^{2} \mathrm{e}^{-V(u)}\right] \in(0, \infty) .
$$

We are interested in the derivative martingale, which is defined as follows:

$$
D_{n}:=\sum_{|u|=n} V(u) \mathrm{e}^{-V(u)} \quad \text { for all } n \geq 0 .
$$

It is a signed martingale with respect to $\left(\mathcal{F}_{n}\right)$, of mean 0 . By [6, Theorem 5.1], under (1.1) and (1.2), $D_{n}$ converges a.s. to a finite nonnegative limit, denoted by $D_{\infty}$. Moreover, $D_{\infty}$ satisfies the following equation (Mandelbrot's cascade):

$$
D_{\infty} \stackrel{\mathrm{D}}{=} \sum_{|u|=1} \mathrm{e}^{-V(u)} D_{\infty}^{(u)},
$$

where $D_{\infty}^{(u)}$ are copies of $D_{\infty}$ independent of each other and $\mathcal{F}_{1}$, and ' $=$, denotes equality in law. Note that $D_{\infty}$ serves as a fixed point of some smoothing transformation. From this point of view, the questions concerning the existence, uniqueness and asymptotic behavior of such fixed points have been much studied in the literature (see [5], [7], [12], [13]). We are interested in the existence of a nontrivial fixed point, and we are going to determine when $\mathbb{P}\left(D_{\infty}>0\right)>0$.

It is known that $\mathbb{P}\left(D_{\infty}=0\right)$ is equal to either the extinction probability $q$ or 1 (see, e.g. [1]). We say that the limit $D_{\infty}$ is nontrivial if $\mathbb{P}\left(D_{\infty}>0\right)>0$, which means that $\mathbb{P}\left(D_{\infty}=0\right)=q$. Otherwise, it is trivially 0 . In this paper, we provide a sufficient and necessary condition for the nontriviality of $D_{\infty}$. The main result is stated as follows.

For any $y \in \mathbb{R}$, let $y_{+}:=\max \{y, 0\}$ and let $\log _{+} y:=\log (\max \{y, 1\})$. We introduce the following random variables:

$$
Y:=\sum_{|u|=1} \mathrm{e}^{-V(u)}, \quad Z:=\sum_{|u|=1} V(u)_{+} \mathrm{e}^{-V(u)} .
$$


Theorem 1.1. The limit of the derivative martingale $D_{n}$ is nontrivial, namely $\mathbb{P}\left(D_{\infty}>0\right)>0$, if and only if the following condition holds:

$$
\mathbb{E}\left[Z \log _{+} Z+Y\left(\log _{+} Y\right)^{2}\right]<\infty .
$$

Remark 1.1. In [6], the authors studied the optimal condition for the nontriviality of $D_{\infty}$. However, there is a small gap between the necessary condition and the sufficient condition for $\mathbb{P}\left(D_{\infty}>0\right)>0$ in their Theorem 5.2. Our result fills this gap and provides the analogue of the result of [18] in the case of branching Brownian motion.

Remark 1.2. Aïdékon proved that (1.3) is sufficient for $\mathbb{P}\left(D_{\infty}>0\right)>0$; see [1, Proposition A.3].

This paper is organized as follows. In Section 2 we introduce a change of measure based on a truncated martingale which is closely related to the derivative martingale. At the end of Section 2 we also prove a proposition concerning certain behaviors of a centered random walk conditioned to stay positive. Then, by using this proposition, we provide the proof of Theorem 1.1 in Section 3.

Throughout the paper, $\left(c_{i}\right)_{i \geq 0}$ denote positive constants. We write $\mathbb{E}[f ; A]$ for $\mathbb{E}\left[f \mathbf{1}_{A}\right]$ and set $\sum_{\varnothing}:=0$.

\section{Lyons' change of measure via truncated martingales}

\subsection{Truncated martingales}

We begin with the well-known many-to-one lemma. For any $a \in \mathbb{R}$, let $\mathbb{P}_{a}$ be the probability measure such that $\mathbb{P}_{a}((V(u), u \in \mathbb{T}) \in \cdot)=\mathbb{P}((V(u)+a, u \in \mathbb{T}) \in \cdot)$. The corresponding expectation is denoted by $\mathbb{E}_{a}$. We write $\mathbb{P}, \mathbb{E}$ instead of $\mathbb{P}_{0}, \mathbb{E}_{0}$ for brevity. For any particle $u \in \mathbb{T}$, we denote by $u_{i}$ its ancestor at the $i$ th generation for $0 \leq i<|u|$. In addition, we write $u_{|u|}:=u$. We thus denote its ancestral line by $\llbracket \varnothing, u \rrbracket:=\left\{u_{0}, u_{1}, \ldots, u_{|u|}\right\}$.

Lemma 2.1. (Many to one.) There exists a sequence of independent and identically distributed (i.i.d.) centered random variables $\left(S_{k+1}-S_{k}\right), k \geq 0$ such that for any $n \geq 1$ and any measurable function $g: \mathbb{R}^{n} \rightarrow \mathbb{R}_{+}$, we have

$$
\mathbb{E}_{a}\left[\sum_{|u|=n} g\left(V\left(u_{1}\right), \ldots, V\left(u_{n}\right)\right)\right]=\mathbb{E}_{a}\left[\mathrm{e}^{S_{n}-a} g\left(S_{1}, \ldots, S_{n}\right)\right]
$$

with $\mathbb{P}_{a}\left[S_{0}=a\right]=1$.

In view of (1.2), $S_{1}-S_{0}$ has the finite variance $\sigma^{2}=\mathbb{E}\left[S_{1}^{2}\right]=\mathbb{E}\left[\sum_{|u|=1} V(u)^{2} \mathrm{e}^{-V(u)}\right]$.

Let $U^{-}(\mathrm{d} y)$ be the renewal measure associated with the weak ascending ladder height process of $\left(-S_{n}, n \geq 0\right)$. Sometimes, we say that $U^{-}(\mathrm{d} y)$ is the renewal measure associated with the weak descending ladder height process of $\left(S_{n}, n \geq 0\right)$. Following the arguments in [4, Section 2], we obtain, for any measurable function $f: \mathbb{R} \rightarrow \mathbb{R}_{+}$,

$$
\mathbb{E}\left[\sum_{j=0}^{\tau-1} f\left(-S_{j}\right)\right]=\int_{0}^{\infty} f(y) U^{-}(\mathrm{d} y),
$$

where $\tau$ is the first time that $\left(S_{n}\right)$ enters $(0, \infty)$, namely $\tau:=\inf \left\{k>0, S_{k} \in(0, \infty)\right\}$ which is proper here. We define $R(x):=U^{-}([0, x))$ for all $x>0$ and define $R(0):=1$. Note that 
$R(x)$ equals the renewal function $U^{-}([0, x])$ at points of continuity. We collect the following properties of this function $R(x)$ which are consequences of the renewal theorem (see [2], [4], [16]).

Lemma 2.2. (i) There exists a positive constant $c_{0}>0$ such that

$$
\lim _{x \rightarrow \infty} \frac{R(x)}{x}=c_{0} .
$$

(ii) There exist two constants $0<c_{1}<c_{2}<\infty$ such that

$$
c_{1}(1+x) \leq R(x) \leq c_{2}(1+x) \text { for all } x \geq 0 .
$$

(iii) For any $x \geq 0$, we have $\mathbb{E}\left[R\left(S_{1}+x\right) \mathbf{1}_{\left\{S_{1}+x>0\right\}}\right]=R(x)$.

Let $\beta \geq 0$. Starting from $V(\varnothing)=a$, we add a barrier at $-\beta$ to the BRW. Now we define the following truncated random variables:

$$
D_{n}^{(\beta)}:=\sum_{|x|=n} R(V(x)+\beta) \mathrm{e}^{-V(x)} \mathbf{1}_{\left\{\min _{1 \leq k \leq n} V\left(x_{k}\right)>-\beta\right\}} \quad \text { for all } n \geq 1,
$$

and $D_{0}^{(\beta)}:=R(a+\beta) \mathrm{e}^{-a} \mathbf{1}_{\{a \geq-\beta\}}$.

Lemma 2.3. For any $a \geq 0$ and $\beta \geq 0$, under $\mathbb{P}_{a}$, the process $\left(D_{n}^{(\beta)}, n \geq 0\right)$ is a nonnegative martingale with respect to $\left(\mathcal{F}_{n}, n \geq 0\right)$.

This lemma follows immediately from Lemma 2.2(iii) and the branching property. We feel free to omit its proof and call $\left(D_{n}^{(\beta)}\right)$ the truncated martingale. It also tells us that under $\mathbb{P}_{a}$, $\left(D_{n}^{(\beta)}, n \geq 0\right)$ converges a.s. to a finite nonnegative limit, which we denote by $D_{\infty}^{(\beta)}$.

The connection between the limits of the derivative martingale and truncated martingales is recorded in the follow Lemma; see [1] and [6] for the proof.

Lemma 2.4. (i) If $D_{\infty}$ is trivial, i.e. $\mathbb{P}\left(D_{\infty}=0\right)=1$, then, for any $\beta \geq 0, D_{\infty}^{(\beta)}$ is trivially 0 under $\mathbb{P}$.

(ii) Under $\mathbb{P}$, if there exists some $\beta \geq 0$ such that $D_{\infty}^{(\beta)}$ is trivially 0 , then so is $D_{\infty}$.

Thanks to Lemma 2.4 , we need only to investigate the truncated martingale $\left(D_{n}^{(0)} ; n \geq 0\right)$ and determine when its limit is nontrivial.

\subsection{Lyons' change of probabilities and spinal decomposition}

Let $\beta=0$. With this nonnegative martingale $\left(D_{n}^{(0)}, n \geq 0\right)$, we define for any $a \geq 0$ a new probability measure $\mathbb{Q}_{a}$ such that, for any $n \geq 1$,

$$
\left.\frac{d \mathbb{Q}_{a}}{d \mathbb{P}_{a}}\right|_{\mathcal{F}_{n}}=\frac{D_{n}^{(0)}}{R(a) \mathrm{e}^{-a}}
$$

where $\mathbb{Q}_{a}$ is defined on $\mathcal{F}_{\infty}:=\vee_{n \geq 0} \mathcal{F}_{n}$. Let us give an intuitive description of the BRW under $\mathbb{Q}_{a}$, which is known as the spinal decomposition. We start from one single particle $\omega_{0}$, located at the position $V\left(\omega_{0}\right)=a$. At time 1, it dies and produces a point process distributed as $(V(u) ;|u|=1)$ under $\mathbb{Q}_{a}$. Among the children of $\omega_{0}, \omega_{1}$ is chosen to be $u$ with probability proportional to $R(V(u)) \mathrm{e}^{-V(u)} \mathbf{1}_{\{V(u)>0\}}$. At each time $n+1$, each particle $v$ in the $n$th generation dies and produces independently a point process distributed as $(V(u) ;|u|=1)$ under $\mathbb{P}_{V(v)}$ 
except $\omega_{n}$, which dies and generates independently a point process distributed as $(V(u) ;|u|=$ 1) under $\mathbb{Q}_{V\left(\omega_{n}\right)}$. And then $\omega_{n+1}$ is chosen to be $u$ among the children of $\omega_{n}$, proportionally to $R(V(u)) \mathrm{e}^{-V(u)} \mathbf{1}_{\left\{\min _{1 \leq k \leq n+1} V\left(u_{k}\right)>0\right\}}$. We still use $\mathbb{T}$ to denote the genealogical tree. Then $\left(\omega_{n} ; n \geq 0\right)$ is an infinite ray in $\mathbb{T}$, which is called the spine. For the rigorous proof, see [1, Appendix A]. Indeed, this type of measure change and the establishment of a spinal decomposition have been developed in various cases of the branching framework; see, e.g. [8], [10], [11], and [14]

We state the following fact about the distribution of the spine process $\left(V\left(\omega_{n}\right), n \geq 0\right)$ under Qa.

Fact 2.1. Let $a \geq 0$. For any $n \geq 0$ and any measurable function $g: \mathbb{R}^{n+1} \rightarrow \mathbb{R}_{+}$, we have

$$
\mathbb{E}_{\mathbb{Q}_{a}}\left[g\left(V\left(\omega_{0}\right), \ldots, V\left(\omega_{n}\right)\right)\right]=\frac{1}{R(a)} \mathbb{E}_{a}\left[g\left(S_{0}, \ldots, S_{n}\right) R\left(S_{n}\right) ; \min _{1 \leq k \leq n} S_{i}>0\right],
$$

where $\left(S_{n}\right)$ is the same as in Lemma 2.1.

For convenience, let $\left(\zeta_{n} ; n \geq 0\right)$ be a stochastic process under $\mathbb{P}_{a}$ such that

$$
\mathbb{P}_{a}\left(\left(\zeta_{n} ; n \geq 0\right) \in \cdot\right)=\mathbb{Q}_{a}\left(\left(V\left(\omega_{n}\right) ; n \geq 0\right) \in \cdot\right) .
$$

Obviously, under $\mathbb{P}_{a},\left(\zeta_{n} ; n \geq 0\right)$ is a Markov chain with transition probabilities $P$ such that, for any $x \geq 0, P(x, \mathrm{~d} y)=(R(y) / R(x)) \mathbf{1}_{\{y>0\}} \mathbb{P}_{x}\left(S_{1} \in \mathrm{d} y\right)$. This process $\zeta_{n}$ is usually called a random walk conditioned to stay positive. It has arisen and been studied in, for example, [2], [4], [16], and [17]. In what follows, we state some results about $\zeta_{n}$, which will be useful later in Section 3.

\subsection{Random walk conditioned to stay positive}

Recall that $\left(S_{n}\right)$ is a centered random walk on $\mathbb{R}$ with finite variance $\sigma^{2}$. Let $\tau_{-}$be the first time that $\left(S_{n}\right)$ hits $(-\infty, 0]$, namely, $\tau_{-}:=\inf \left\{k \geq 1: S_{k} \leq 0\right\}$. Let $\left(T_{k}, H_{k} ; k \geq 0\right)$ be the strict ascending ladder epochs and heights of $\left(S_{n} ; n \geq 0\right)$, i.e. $T_{0}=0, H_{0}:=S_{0}$, and for any $k \geq 1, T_{k}:=\inf \left\{j>T_{k-1}: S_{j}>H_{k-1}\right\}, H_{k}:=S_{T_{k}}$. We denote by $U(\mathrm{~d} x)$ the corresponding renewal measure (see, e.g. [9, Chapter XII]). Then, similarly to (2.1), for any measurable function $f: \mathbb{R} \rightarrow \mathbb{R}_{+}$,

$$
\mathbb{E}\left[\sum_{n=0}^{\tau_{-}-1} f\left(S_{n}\right)\right]=\int_{0}^{\infty} f(x) U(\mathrm{~d} x) .
$$

From (2.3) and (2.5), we deduce

$$
\begin{aligned}
\mathbb{E}\left[\sum_{n \geq 0} f\left(\zeta_{n}\right)\right] & =\mathbb{E}_{\mathbb{Q}_{0}}\left[\sum_{n \geq 0} f\left(V\left(\omega_{n}\right)\right)\right] \\
& =\sum_{n \geq 0} \mathbb{E}\left[f\left(S_{n}\right) R\left(S_{n}\right) \mathbf{1}_{\left\{\min _{1 \leq k \leq n} S_{k}>0\right\}}\right] \\
& =\mathbb{E}\left[\sum_{n=0}^{\tau_{-}-1} f\left(S_{n}\right) R\left(S_{n}\right)\right] \\
& =\int_{0}^{\infty} f(x) R(x) U(\mathrm{~d} x)
\end{aligned}
$$


Recall also that $U^{-}(\mathrm{d} x)$ is the renewal measure associated with the weak descending ladder height process of $\left(S_{n}\right)$. By the renewal theorem (see [9, p. 360]), there exist two constants $c_{3}$, $c_{4}>0$ such that for all $x, y \geq 0$,

$$
\begin{array}{cc}
c_{3}(1+x) \leq U([0, x]) \leq c_{4}(1+x), & 0 \leq U([x, x+y]) \leq c_{4}(1+y), \\
c_{3}(1+x) \leq U^{-}([0, x]) \leq c_{4}(1+x), & 0 \leq U^{-}([x, x+y]) \leq c_{4}(1+y) .
\end{array}
$$

Given a nonincreasing and positive function $F$, we present the following proposition, which provides a necessary and sufficient condition for the infinity of the series $\sum_{n} F\left(\zeta_{n}\right)$.

Proposition 2.1. Let $F:[0, \infty) \rightarrow[0, \infty)$ be nonincreasing. Then

$$
\int_{0}^{\infty} F(y) y \mathrm{~d} y=\infty \Longleftrightarrow \sum_{n \geq 0} F\left(\zeta_{n}\right)=\infty, \quad \mathbb{P} \text {-a.s. }
$$

Note that $\left(\zeta_{n}\right)$ can be viewed as a discrete-time counterpart of the Bessel process, for which a similar result holds (see, e.g. [15, Example 2.5, Chapter XI]). We will prove (2.9) in a similar way as for the Bessel process.

Proof. Observe that $0 \leq F(x) \leq F(0)<\infty$ for any $x \geq 0$. So there is no difference between the two events $\left\{\sum_{n \geq 0} F\left(\zeta_{n}\right)=\infty\right\}$ and $\left\{\sum_{n \geq 1} F\left(\zeta_{n}\right)=\infty\right\}$.

We first prove ' $\Longleftarrow$ ' in (2.9). From (2.2) and (2.7), it follows that

$$
\int_{0}^{\infty} F(y) y \mathrm{~d} y=\infty \quad \Longleftrightarrow \int_{0}^{\infty} F(y) R(y) U(\mathrm{~d} y)=\infty .
$$

Actually, by (2.6),

$$
\mathbb{E}\left[\sum_{n \geq 0} F\left(\zeta_{n}\right)\right]=\int_{0}^{\infty} F(y) R(y) U(\mathrm{~d} y) .
$$

Clearly, $\mathbb{P}\left(\sum_{n \geq 0} F\left(\zeta_{n}\right)=\infty\right)=1$ yields $\int_{0}^{\infty} F(y) R(y) U(\mathrm{~d} y)=\infty$. The ' $\Longleftarrow$ ' in (2.9) is, hence, proved.

To prove ' $\Longrightarrow$ ' in (2.9), we need only to show that if $\mathbb{P}\left(\sum_{n>0} F\left(\zeta_{n}\right)=\infty\right)<1$, then $\int_{0}^{\infty} F(y) y \mathrm{~d} y<\infty$. From now on, we suppose that $\mathbb{P}\left(\sum_{n \geq 0} F\left(\zeta_{n}\right)=\infty\right)<1$, which is equivalent to

$$
\mathbb{P}\left(\sum_{n \geq 1} F\left(\zeta_{n}\right)<\infty\right)>0
$$

We draw support from Tanaka's construction for the random walk conditioned to stay positive (see [16], [4]). Recall that $\tau=\inf \left\{k \geq 1: S_{k} \in(0, \infty)\right\}$. Hence, we obtain an excursion $\left(S_{j} ; 0 \leq j \leq \tau\right)$, which is denoted by $\xi=(\xi(j), 0 \leq j \leq \tau)$. Let $\left\{\xi_{k}=\left(\xi_{k}(j), 0 \leq j \leq\right.\right.$ $\left.\left.\tau_{k}\right) ; k \geq 1\right\}$ be a sequence of independent copies of $\xi$. For any $k \geq 1$, let

$$
\nu_{k}(j):=\xi_{k}\left(\tau_{k}\right)-\xi_{k}\left(\tau_{k}-j\right) \text { for all } 0 \leq j \leq \tau_{k} .
$$

This brings out another sequence of i.i.d. excursions $\left\{v_{k}=\left(v_{k}(j), ; 0 \leq j \leq \tau_{k}\right) ; k \geq 1\right\}$, based on which we reconstruct the random walk conditioned to stay positive $\left(\zeta_{n}\right)$ in the following way. Define, for any $k \geq 1$,

$$
T_{k}^{+}:=\tau_{1}+\cdots+\tau_{k}, \quad H_{k}^{+}:=v_{1}\left(\tau_{1}\right)+\cdots+v_{k}\left(\tau_{k}\right)=\xi_{1}\left(\tau_{1}\right)+\cdots+\xi_{k}\left(\tau_{k}\right),
$$

and let $T_{0}^{+}=H_{0}^{+}=0$. Then the process

$$
\zeta_{n}=H_{k}^{+}+v_{k+1}\left(n-T_{k}^{+}\right) \quad \text { for } T_{k}^{+}<n \leq T_{k+1}^{+}
$$

with $\zeta_{0}=0$, is what we need. 
We actually establish a process distributed as $\left(\zeta_{n}\right)$. For brevity, we still denote it by $\left(\zeta_{n}\right)$ without changing any conclusion in this proof. For any $k \geq 1$, let

$$
\chi_{k}(F):=\sum_{n=T_{k-1}^{+}+1}^{T_{k}^{+}} F\left(\zeta_{n}\right)=\sum_{j=1}^{\tau_{k}} F\left(H_{k-1}^{+}+v_{k}(j)\right)
$$

so $\sum_{n \geq 1} F\left(\zeta_{n}\right)=\sum_{k \geq 1} \chi_{k}(F)$.

On the other hand, by (2.11),

$$
\chi_{k}(F)=\sum_{j=1}^{\tau_{k}} F\left(H_{k-1}^{+}+\xi_{k}\left(\tau_{k}\right)-\xi_{k}\left(\tau_{k}-j\right)\right)=\sum_{j=0}^{\tau_{k}-1} F\left(H_{k}^{+}-\xi_{k}(j)\right) .
$$

Hence, (2.10) becomes

$$
\mathbb{P}\left(\sum_{k \geq 1} \chi_{k}(F)<\infty\right)=\mathbb{P}\left(\sum_{k \geq 1} \sum_{j=0}^{\tau_{k}-1} F\left(H_{k}^{+}-\xi_{k}(j)\right)<\infty\right)>0 .
$$

By [9, Theorem 1, Chapter XVIII.5], as $\left(S_{n}\right)$ is of finite variance, we have $b^{+}:=\mathbb{E}\left[H_{1}^{+}\right]<\infty$. It follows from the strong law of large numbers that $\mathbb{P}$-a.s.,

$$
\lim _{k \rightarrow \infty} \frac{H_{k}^{+}}{k}=b^{+}
$$

Let $\delta>\left(1-b^{+}\right)_{+}$. This tells us that $\mathbb{P}$-a.s. for all large $k, H_{k}^{+} \leq\left(b^{+}+\delta\right) k$. As $F$ is nonincreasing, we have

$$
\mathbb{P}\left(\sum_{k \geq 1} \sum_{j=0}^{\tau_{k}-1} F\left(k\left(b^{+}+\delta\right)-\xi_{k}(j)\right)<\infty\right) \geq \mathbb{P}\left(\sum_{k \geq 1} \sum_{j=0}^{\tau_{k}-1} F\left(H_{k}^{+}-\xi_{k}(j)\right)<\infty\right)>0
$$

Let $A:=b^{+}+\delta$ and for any $k \geq 1$ let

$$
\tilde{\chi}_{k}:=\sum_{j=0}^{\tau_{k}-1} F\left(A k-\xi_{k}(j)\right)
$$

So $\mathbb{P}\left(\sum_{k \geq 1} \tilde{\chi}_{k}<\infty\right)>0$. Recall that $\left\{\xi_{k}, k \geq 1\right\}$ is a sequence of independent copies of $\left(S_{j} ; 0 \leq j \leq \tau\right)$. This yields the independence of the sequence $\left\{\tilde{\chi}_{k}, k \geq 1\right\}$. From Kolmogorov's 0-1 law, it follows that

$$
\mathbb{P}\left[\sum_{k \geq 1} \sum_{j=0}^{\tau_{k}-1} F\left(A k-\xi_{k}(j)\right)<\infty\right]=\mathbb{P}\left[\sum_{k \geq 1} \tilde{\chi}_{k}<\infty\right]=1
$$

Moreover, let $E_{M}:=\left\{\sum_{k \geq 1} \tilde{\chi}_{k}<M\right\}$ for any $M>0$. Either there exists some $M_{0}<\infty$ such that $\mathbb{P}\left[E_{M_{0}}\right]=1$, or $\mathbb{P}\left(E_{M}\right)<1$ for all $M \in(0, \infty)$. On the one hand, if $\mathbb{P}\left(E_{M_{0}}\right)=1$ 
for some $M_{0}<\infty$, then

$$
\begin{aligned}
M_{0} & \geq \mathbb{E}\left[\sum_{k \geq 1} \tilde{\chi}_{k}\right] \\
& =\mathbb{E}\left[\sum_{k \geq 1} \sum_{j=0}^{\tau_{k}-1} F\left(A k-\xi_{k}(j)\right)\right] \\
& =\sum_{k \geq 1} \mathbb{E}\left[\sum_{j=0}^{\tau-1} F\left(A k-S_{j}\right)\right] \\
& =\sum_{k \geq 1} \int_{0}^{\infty} F(A k+y) U^{-}(\mathrm{d} y),
\end{aligned}
$$

where the last equality follows from (2.5). We see that $\sum_{k \geq 1} \int_{0}^{\infty} F(A k+y) U^{-}(\mathrm{d} y)<\infty$. It follows from the renewal theorem that there exists $B>0$ such that $U^{-}([j B, j B+B])>\delta>0$ for any $j \geq 0$. As $F$ is nonincreasing,

$$
\frac{\delta}{B} \int_{A+B}^{\infty} F(x) \mathrm{d} x \leq \sum_{j \geq 1} F(A+B j) \delta \leq \int_{0}^{\infty} F(A+y) U^{-}(\mathrm{d} y)<\infty
$$

which implies that $\int_{0}^{\infty} F(x) \mathrm{d} x<\infty$. Moreover,

$$
\sum_{k \geq 1} \sum_{j \geq 1} F(A k+B j) \delta \leq \sum_{k \geq 1} \int_{0}^{\infty} F(A k+y) U^{-}(\mathrm{d} y)<\infty .
$$

Hence, we observe that $\int_{A}^{\infty} \mathrm{d} z \int_{B}^{\infty} F(y+z) \mathrm{d} y \leq \sum_{k \geq 1} \sum_{j \geq 1} F(A k+B j) A B<\infty$. This implies that

$$
\begin{aligned}
\int_{0}^{\infty} F(x) x \mathrm{~d} x & =\int_{0}^{\infty} \mathrm{d} z \int_{0}^{\infty} F(z+y) \mathrm{d} y \\
& \leq F(0) A B+A \int_{B}^{\infty} F(x) \mathrm{d} x+B \int_{A}^{\infty} F(x) \mathrm{d} x+\int_{A}^{\infty} \mathrm{d} z \int_{B}^{\infty} F(y+z) \mathrm{d} y \\
& <\infty
\end{aligned}
$$

which is what we need.

On the other hand, if $\mathbb{P}\left(E_{M}\right)<1$ for all $M \in(0, \infty)$, we have $\lim _{M \uparrow \infty} \mathbb{P}\left(E_{M}\right)=1$ due to (2.12). For any $k \geq 1$ and any $\ell \geq 1$, define

$$
\Lambda_{\ell}^{(k)}:=\sum_{j=0}^{\tau_{k}-1} \mathbf{1}_{\left\{A(\ell-1) \leq-\xi_{k}(j)<A \ell\right\}}
$$


As $\sum_{\ell \geq 1} \mathbf{1}_{\left\{A(\ell-1) \leq-\xi_{k}(j)<A \ell\right\}}=1$, we obtain, for any $k \geq 1$,

$$
\begin{aligned}
\tilde{\chi}_{k} & =\sum_{j=0}^{\tau_{k}-1} F\left(A k-\xi_{k}(j)\right) \sum_{\ell \geq 1} \mathbf{1}_{\left\{A(\ell-1) \leq-\xi_{k}(j)<A \ell\right\}} \\
& =\sum_{\ell \geq 1} \sum_{j=0}^{\tau_{k}-1} F\left(A k-\xi_{k}(j)\right) \mathbf{1}_{\left\{A(\ell-1) \leq-\xi_{k}(j)<A \ell\right\}} \\
& \geq \sum_{\ell \geq 1} F(A k+A \ell) \Lambda_{\ell}^{(k)},
\end{aligned}
$$

where the last inequality holds because $F$ is nonincreasing. It follows that

$$
\begin{aligned}
\sum_{k \geq 1} \tilde{\chi}_{k} & \geq \sum_{k \geq 1} \sum_{\ell \geq 1} F(A k+A \ell) \Lambda_{\ell}^{(k)} \\
& =\sum_{n=2}^{\infty} F(A n) \sum_{k=1}^{n-1} \Lambda_{n-k}^{(k)} \\
& =\sum_{m=1}^{\infty} F(A m+A) m Y_{m},
\end{aligned}
$$

where $Y_{m}:=\sum_{k=1}^{m} \Lambda_{m+1-k}^{(k)} / m$ for all $m \geq 1$. We claim that there exists a $M>0$ sufficiently large such that, for any $m \geq 1$,

$$
c_{6} \geq \mathbb{E}\left[Y_{m} \mathbf{1}_{E_{M}}\right] \geq c_{5}>0,
$$

where $c_{5}, c_{6}$ are positive constants. We postpone the proof of (2.14) and return to (2.13). It follows that

$$
\begin{aligned}
M & \geq \mathbb{E}\left[\mathbf{1}_{E_{M}} \sum_{k \geq 1} \tilde{\chi}_{k}\right] \\
& \geq \mathbb{E}\left[\mathbf{1}_{E_{M}} \sum_{m=1}^{\infty} F(A m+A) m Y_{m}\right] \\
& \geq \sum_{m \geq 1} F(A m+A) m \mathbb{E}\left[Y_{m} \mathbf{1}_{E_{M}}\right] .
\end{aligned}
$$

By (2.14), we obtain

$$
\sum_{m \geq 1} F(A m+A) m \leq \frac{M}{c_{5}}<\infty .
$$

This implies that $\int_{0}^{\infty} F(y) y \mathrm{~d} y<\infty$, which completes the proof of Proposition (2.1).

It remains to prove (2.14).

We begin with the first and second moments of $Y_{m}$. Since $\left\{\xi_{k}, k \geq 1\right\}$ are i.i.d. copies of $\left(S_{j}, 0 \leq j \leq \tau\right),\left\{\left(\Lambda_{\ell}^{(k)} ; \ell \geq 1\right), k \geq 1\right\}$ are i.i.d. This yields

$$
\begin{aligned}
\mathbb{E}\left[Y_{m}\right] & =\frac{1}{m} \sum_{k=1}^{m} \mathbb{E}\left[\Lambda_{m+1-k}^{(k)}\right] \\
& =\frac{1}{m} \sum_{k=1}^{m} \mathbb{E}\left[\Lambda_{m+1-k}^{(1)}\right]
\end{aligned}
$$




$$
\begin{aligned}
& =\frac{1}{m} \mathbb{E}\left[\sum_{k=1}^{m} \Lambda_{k}^{(1)}\right] \\
& =\frac{1}{m} \mathbb{E}\left[\sum_{j=0}^{\tau-1} \mathbf{1}_{\left\{-S_{j}<A m\right\}}\right] \\
& =\frac{R(A m)}{m}
\end{aligned}
$$

where the last equality comes from (2.1). By (2.2), for any $m \geq 1$,

$$
c_{1} A \leq \mathbb{E}\left[Y_{m}\right] \leq c_{2}(A+1)=: c_{6} .
$$

Obviously, we have $\mathbb{E}\left[Y_{m} \mathbf{1}_{E_{M}}\right] \leq c_{6}$ for any $m \geq 1$. The fact that $\Lambda^{(k)}, k \geq 1$, are i.i.d. yields also

$$
\operatorname{var}\left(Y_{m}\right)=\frac{1}{m^{2}} \sum_{k=1}^{m} \operatorname{var}\left(\Lambda_{k}^{(1)}\right) \leq \frac{1}{m^{2}} \sum_{k=1}^{m} \mathbb{E}\left[\left(\Lambda_{k}^{(1)}\right)^{2}\right] .
$$

Note that $\Lambda_{1}^{(1)}$ is distributed as $\sum_{j=0}^{\tau-1} \mathbf{1}_{\left\{-S_{j}<A\right\}}$ with $\tau=\inf \left\{k>0 ; S_{k}>0\right\}$. We have

$$
\mathbb{E}\left[\left(\Lambda_{1}^{(1)}\right)^{2}\right]=\mathbb{E}\left[\left(\sum_{j=0}^{\tau-1} \mathbf{1}_{\left\{-S_{j}<A\right\}}\right)^{2}\right] \leq 2 \mathbb{E}\left[\sum_{j=0}^{\tau-1} \mathbf{1}_{\left\{-S_{j}<A\right\}} \sum_{k=j}^{\tau-1} \mathbf{1}_{\left\{-S_{k}<A\right\}}\right] .
$$

By the Markov property, we obtain

$$
\mathbb{E}\left[\left(\Lambda_{1}^{(1)}\right)^{2}\right] \leq 2 \mathbb{E}\left[\sum_{j=0}^{\tau-1} \mathbf{1}_{\left\{-S_{j}<A\right\}} R\left(A,-S_{j}\right)\right]
$$

where

$$
R(x, y):=\mathbb{E}\left[\sum_{i=0}^{\tau_{y}-1} \mathbf{1}_{\left\{S_{i}>y-x\right\}}\right]
$$

with $\tau_{y}:=\inf \left\{k>0 ; S_{k}>y\right\}$ for $x, y \geq 0$. From (2.1), it follows that

$$
\mathbb{E}\left[\left(\Lambda_{1}^{(1)}\right)^{2}\right] \leq 2 \int_{0}^{A} R(A, y) U^{-}(\mathrm{d} y) .
$$

Consider now the strict ascending ladder epochs and heights $\left(T_{k}, H_{k}\right)$ of $\left(S_{n}\right)$. We obtain

$$
R(x, y)=\mathbb{E}\left[\sum_{k=0}^{\infty} \mathbf{1}_{\left\{y \geq H_{k}>y-x\right\}} \sum_{n=T_{k}}^{T_{k+1}-1} \mathbf{1}_{\left\{S_{n}>y-x\right\}}\right] .
$$

By applying the Markov property at the times $T_{k}$, and (2.1), we have for $x, y \geq 0$,

$$
R(x, y)=\mathbb{E}\left[\sum_{k \geq 0} R\left(H_{k}+x-y\right) \mathbf{1}_{\left\{y \geq H_{k}>y-x\right\}}\right]=\int_{(y-x)_{+}}^{y} R(x-y+z) U(\mathrm{~d} z),
$$

Substituting (2.18) into (2.17) and then using (2.2), (2.8) and using (2.7) implies that

$$
\mathbb{E}\left[\left(\Lambda_{1}^{(1)}\right)^{2}\right] \leq c_{7}(1+A)^{3} \leq c_{8} A^{3} ;
$$

see also [4, Lemma 2]. 
Moreover, for any $\ell \geq 2, \Lambda_{\ell}^{(1)}$ has the same law as $\sum_{j=0}^{\tau-1} \mathbf{1}_{\left\{\ell A-A \leq-S_{j}<\ell A\right\}}$. Similarly, we obtain

$$
\begin{aligned}
\mathbb{E}\left[\left(\Lambda_{\ell}^{(1)}\right)^{2}\right] & =\mathbb{E}\left[\left(\sum_{j=0}^{\tau-1} \mathbf{1}_{\left\{\ell A-A \leq-S_{j}<\ell A\right\}}\right)^{2}\right] \\
& \leq 2 \mathbb{E}\left[\sum_{j=0}^{\tau-1} \mathbf{1}_{\left\{\ell A-A \leq-S_{j}<\ell A\right\}} \sum_{k=j}^{\tau-1} \mathbf{1}_{\left\{\ell A-A \leq-S_{k}<\ell A\right\}}\right] .
\end{aligned}
$$

Once again, by the Markov property and then by (2.1), we have

$$
\begin{aligned}
\mathbb{E}\left[\left(\Lambda_{\ell}^{(1)}\right)^{2}\right] & \leq 2 \mathbb{E}\left[\sum_{j=0}^{\tau-1} \mathbf{1}_{\left\{\ell A-A \leq-S_{j}<\ell A\right\}}\left(R\left(\ell A,-S_{j}\right)-R\left(\ell A-A,-S_{j}\right)\right)\right] \\
& =2 \int_{\ell A-A}^{\ell A}(R(\ell A, y)-R(\ell A-A, y)) U^{-}(\mathrm{d} y) .
\end{aligned}
$$

Substituting (2.18) into this equation yields for $\ell \geq 2$,

$\mathbb{E}\left[\left(\Lambda_{\ell}^{(1)}\right)^{2}\right]$

$$
\begin{gathered}
\leq 2 \int_{\ell A-A}^{\ell A}\left(\int_{0}^{y} R(\ell A-y+z) U(\mathrm{~d} z)-\int_{y-\ell A+A}^{y} R(\ell A-A-y+z) U(\mathrm{~d} z)\right) U^{-}(\mathrm{d} y) \\
=2 \int_{\ell A-A}^{\ell A}\left(\int_{0}^{y-\ell A+A} R(\ell A-y+z) U(\mathrm{~d} z)\right. \\
\left.\quad+\int_{y-\ell A+A}^{y} U^{-}([\ell A-A-y+z, \ell A-y+z)) U(\mathrm{~d} z)\right) U^{-}(\mathrm{d} y),
\end{gathered}
$$

where the last equality holds because $R(x)=U^{-}([0, x))$. Observe that $R(\ell A-y+z) \leq R(A)$ for $0 \leq z \leq y-\ell A+A$ and $\ell A-A \leq y \leq \ell A$. Recall that $A \geq 1$. By (2.2), (2.7), and (2.8),

$$
\begin{aligned}
\mathbb{E}\left[\left(\Lambda_{\ell}^{(1)}\right)^{2}\right] & \leq c_{9} \int_{0}^{A}\left(\int_{0}^{u}(A-u+z+1) U(\mathrm{~d} z)+\int_{u}^{u+(\ell-1) A}(1+A) U(\mathrm{~d} z)\right) U^{-}(\mathrm{d} u) \\
& \leq c_{10}(A+1) \int_{0}^{A}(u+1+\ell A) U^{-}(\mathrm{d} u) \\
& \leq c_{11} \ell A^{3}
\end{aligned}
$$

with $c_{11} \geq c_{8}$. Returning to (2.16), for any $m \geq 1$,

$$
\operatorname{var}\left(Y_{m}\right) \leq \frac{\sum_{\ell=1}^{m} c_{11} \ell A^{3}}{m^{2}} \leq c_{12} A^{3}
$$

Combining this with (2.15) implies that $\mathbb{E}\left[Y_{m}^{2}\right]=\operatorname{var}\left(Y_{m}\right)+\mathbb{E}\left[Y_{m}\right]^{2} \leq c_{2}^{2}(1+A)^{2}+c_{12} A^{3}$. We then use the Paley-Zygund inequality to obtain

$$
\mathbb{P}\left(Y_{m}>\frac{1}{2} \mathbb{E}\left[Y_{m}\right]\right) \geq \frac{\mathbb{E}\left[Y_{m}\right]^{2}}{4 \mathbb{E}\left[Y_{m}^{2}\right]} \geq \frac{c_{1}^{2} A^{2}}{4\left(c_{2}^{2}(1+A)^{2}+c_{12} A^{3}\right)}:=c_{13}>0 .
$$


So, for any $0 \leq u \leq c_{1} A / 2 \leq \mathbb{E}\left[Y_{m}\right] / 2$, we have

$$
\mathbb{P}\left(Y_{m} \leq u\right) \leq \mathbb{P}\left(Y_{m} \leq \frac{\mathbb{E}\left[Y_{m}\right]}{2}\right) \leq 1-c_{13} .
$$

There exists $M_{1}>0$ such that $\mathbb{P}\left(E_{M_{1}}\right) \geq 1-c_{13} / 2$, since $\lim _{M \uparrow \infty} \mathbb{P}\left[E_{M}\right]=1$. For such $M_{1}>0$,

$$
\mathbb{E}\left[Y_{m} \mathbf{1}_{E_{M_{1}}}\right]=\mathbb{E}\left[\int_{0}^{Y_{m}} \mathbf{1}_{E_{M_{1}}} \mathrm{~d} u\right]=\int_{0}^{\infty} \mathbb{P}\left[Y_{m}>u ; E_{M_{1}}\right] \mathrm{d} u .
$$

Note that $\mathbb{P}\left(Y_{m}>u, E_{M_{1}}\right) \geq\left(\mathbb{P}\left(E_{M_{1}}\right)-\mathbb{P}\left[Y_{m} \leq u\right]\right)_{+}$, which is greater than $c_{13} / 2$ when $0 \leq u \leq c_{1} A / 2$. As a consequence,

$$
\mathbb{E}\left[Y_{m} \mathbf{1}_{E_{M_{1}}}\right]=\int_{0}^{\infty} \mathbb{P}\left(Y_{m}>u ; E_{M_{1}}\right) \mathrm{d} u \geq \int_{0}^{c_{1} A / 2} \frac{c_{13}}{2} \mathrm{~d} u=\frac{c_{1} c_{13} A}{4}=: c_{5}>0 .
$$

This completes the proof of (2.14), and hence completes the proof of ' $\Longrightarrow$ ' in (2.9). Proposition 2.1 is now proved.

\section{Proof of the main theorem}

Recall that we are in the following regime:

$$
\begin{gathered}
\mathbb{E}\left[\sum_{|u|=1} \mathrm{e}^{-V(u)}\right]=1, \quad \mathbb{E}\left[\sum_{|u|=1} V(u) \mathrm{e}^{-V(u)}\right]=0, \\
\sigma^{2}=\mathbb{E}\left[\sum_{|u|=1} V(u)^{2} \mathrm{e}^{-V(u)}\right]<\infty .
\end{gathered}
$$

Recall also that equivalence in Theorem 1.1 is as follows:

$$
\mathbb{E}\left[Y\left(\log _{+} Y\right)^{2}\right]+\mathbb{E}\left[Z \log _{+} Z\right]<\infty \quad \Longleftrightarrow \mathbb{P}\left(D_{\infty}>0\right)>0
$$

with $Y=\sum_{|u|=1} \mathrm{e}^{-V(u)}$ and $Z=\sum_{|u|=1} V(u)_{+} \mathrm{e}^{-V(u)}$.

This section is devoted to proving that the condition on the left-hand side of (3.2) (i.e. (1.3)) is a necessary and sufficient condition for mean convergence of the truncated martingale

$$
\left\{D_{n}^{(0)}=\sum_{|u|=n} R(V(u)) \mathrm{e}^{-V(u)} \mathbf{1}_{\left\{V\left(u_{k}\right)>0 \text { for all } 1 \leq k \leq n\right\}} ; n \geq 0\right\} .
$$

In view of Lemma 2.4, the nontriviality of $D_{\infty}$ follows, which proves Theorem 1.1.

In what follows, we state a result concerning the mean convergence of the truncated martingale $\left\{D_{n}^{(0)} ; n \geq 0\right\}$, which is a special case of [6, Theorem 2.1].

Define

$$
X:=\frac{D_{1}^{(0)}}{D_{0}^{(0)}} \mathbf{1}_{\left\{D_{0}^{(0)}>0\right\}}+\mathbf{1}_{\left\{D_{0}^{(0)}=0\right\}} .
$$

Then for any $a \geq 0$, under $\mathbb{P}_{a}$,

$$
X=\frac{\sum_{|u|=1} R(V(u)) \mathrm{e}^{-V(u)} \mathbf{1}_{\{V(u)>0\}}}{R(a) \mathrm{e}^{-a}} .
$$


Theorem 3.1. (See Biggins and Kyprianou [6].) Let $\left(\zeta_{n}\right)$ be a random walk conditioned to stay positive, whose law was given in (2.4).

(i) If

$$
\sum_{n \geq 1} \mathbb{E}_{\zeta_{n}}\left[X\left(R\left(\zeta_{n}\right) \mathrm{e}^{-\zeta_{n}} X \wedge 1\right)\right]<\infty, \quad \mathbb{P} \text {-a.s. }
$$

then $\mathbb{E}\left[D_{\infty}^{(0)}\right]=R(0)$.

(ii) If for all $y>0$,

$$
\sum_{n=1}^{\infty} \mathbb{E}_{\zeta_{n}}\left[X ; R\left(\zeta_{n}\right) \mathrm{e}^{-\zeta_{n}} X \geq y\right]=\infty, \quad \mathbb{P} \text {-a.s. }
$$

then $\mathbb{E}\left[D_{\infty}^{(0)}\right]=0$.

Our proof relies on this theorem. First, in Section 3.1 we provide a short proof for the sufficient part to accomplish our arguments even though it has already been proved in [1]. In Section 3.2 we prove that (1.8) is also the necessary condition by using Proposition 2.1.

\subsection{Equation (1.3) is a sufficient condition}

This section is devoting to proving that

$$
\mathbb{E}\left[Y\left(\log _{+} Y\right)^{2}\right]+\mathbb{E}\left[Z \log _{+} Z\right]<\infty \quad \Longrightarrow \quad \mathbb{E}\left[D_{\infty}^{(0)}\right]=R(0)=1 .
$$

Proof of (3.4). According to Theorem 3.1(i), it suffices to show that

$\mathbb{E}\left[Y\left(\log _{+} Y\right)^{2}\right]+\mathbb{E}\left[Z \log _{+} Z\right]<\infty \quad \Longrightarrow \quad \sum_{n \geq 0} \mathbb{E}_{\zeta_{n}}\left[X\left(R\left(\zeta_{n}\right) \mathrm{e}^{-\zeta_{n}} X \wedge 1\right)\right]<\infty, \quad \mathbb{P}$-a.s.

For any particle $x \in \mathbb{T} \backslash\{\varnothing\}$, we denote its parent by $\overleftarrow{u}$ and define its relative displacement by

$$
\Delta V(u):=V(u)-V(\overleftarrow{u})
$$

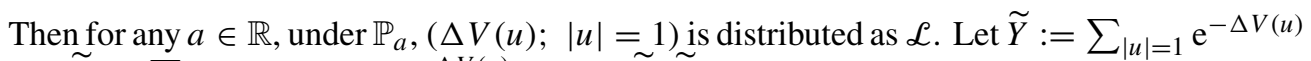
and $\widetilde{Z}:=\sum_{|u|=1}(\Delta V(u))_{+} \mathrm{e}^{-\Delta V(u)}$, so $\mathbb{P}_{a}[(\widetilde{Y}, \widetilde{Z}) \in \cdot]=\mathbb{P}((Y, Z) \in \cdot)$.

Note that under $\mathbb{P}_{\zeta_{n}}$,

$$
\begin{aligned}
X & =\frac{\sum_{|u|=1} R(V(u)) \mathrm{e}^{-V(u)} \mathbf{1}_{\{V(u)>0\}}}{R\left(\zeta_{n}\right) \mathrm{e}^{-\zeta_{n}}} \\
& =\frac{\sum_{|u|=1} R\left(\zeta_{n}+\Delta V(u)\right) \mathrm{e}^{-\Delta V(u)} \mathbf{1}_{\left\{\Delta V(u)>-\zeta_{n}\right\}}}{R\left(\zeta_{n}\right)},
\end{aligned}
$$


where $(\Delta V(u) ;|u|=1)$ is independent of $\zeta_{n}$. By (2.2), it follows that

$$
\begin{aligned}
X & \leq \frac{\sum_{|u|=1} c_{2}\left(\zeta_{n}+1\right) \mathrm{e}^{-\Delta V(u)} \mathbf{1}_{\left\{\Delta V(u)>-\zeta_{n}\right\}}}{R\left(\zeta_{n}\right)}+\frac{\sum_{|u|=1} c_{2} \Delta V(u) \mathrm{e}^{-\Delta V(u)} \mathbf{1}_{\left\{\Delta V(u)>-\zeta_{n}\right\}}}{R\left(\zeta_{n}\right)} \\
& \leq \frac{c_{2}}{c_{1}} \sum_{|u|=1} \mathrm{e}^{-\Delta V(u)}+c_{2} \frac{\sum_{|u|=1} \Delta V(u)_{+} \mathrm{e}^{-\Delta V(u)}}{R\left(\zeta_{n}\right)} \\
& \leq c_{14}\left(\tilde{Y}+\frac{\widetilde{Z}}{R\left(\zeta_{n}\right)}\right) \\
& \leq 2 c_{14} \max \left\{\tilde{Y}, \frac{\widetilde{Z}}{R\left(\zeta_{n}\right)}\right\}
\end{aligned}
$$

where $(\tilde{Y}, \widetilde{Z})$ is independent of $\zeta_{n}$. This implies that

$$
\begin{aligned}
& \sum_{n \geq 1} \mathbb{E}_{\zeta_{n}}\left[X\left(R\left(\zeta_{n}\right) \mathrm{e}^{-\zeta_{n}} X \wedge 1\right)\right] \\
& \quad \leq c_{15}\left(\sum_{n \geq 0} \mathbb{E}\left[\tilde{Y}\left(R\left(\zeta_{n}\right) \mathrm{e}^{-\zeta_{n}} \tilde{Y} \wedge 1\right) \mid \zeta_{n}\right]+\sum_{n \geq 0} \frac{1}{R\left(\zeta_{n}\right)} \mathbb{E}\left[\widetilde{Z}\left(\mathrm{e}^{-\zeta_{n}} \widetilde{Z} \wedge 1\right) \mid \zeta_{n}\right]\right) \\
& \quad=: c_{15}\left(\Sigma_{1}+\Sigma_{2}\right) .
\end{aligned}
$$

Hence, we need only to prove that

$$
\mathbb{E}\left[Y\left(\log _{+} Y\right)^{2}\right]+\mathbb{E}\left[Z \log _{+} Z\right]<\infty \quad \Longrightarrow \quad \mathbb{E}\left[\Sigma_{1}\right]+\mathbb{E}\left[\Sigma_{2}\right]<\infty,
$$

which leads to (3.5). On the one hand, from (2.2) it follows that $R(x) \leq c_{21} \mathrm{e}^{x / 2}$ for all $x \geq 0$, so we have

$$
\begin{aligned}
\mathbb{E}\left[\Sigma_{1}\right] & \leq c_{17} \mathbb{E}\left[\sum_{n \geq 0} \mathbb{E}\left[\tilde{Y}\left(\mathrm{e}^{-\zeta_{n} / 2} \tilde{Y} \wedge 1\right) \mid \zeta_{n}\right]\right] \\
& =c_{17} \sum_{n \geq 0} \mathbb{E}\left[(\tilde{Y})^{2} \mathrm{e}^{-\zeta_{n}} \mathbf{1}_{\left\{\tilde{Y} \leq \mathrm{e}^{\left.\zeta_{n} / 2\right\}}\right.}+\tilde{Y} \mathbf{1}_{\left\{\tilde{Y}>\mathrm{e}^{\left.\zeta_{n} / 2\right\}}\right]}\right] \\
& =c_{17} \mathbb{E}\left\{(\tilde{Y})^{2} \mathbb{E}\left[\sum_{n \geq 0} \mathrm{e}^{-\zeta_{n}} \mathbf{1}_{\left\{\zeta_{n} \geq 2 \log \tilde{Y}\right\}} \mid \tilde{Y}\right]+\tilde{Y} \mathbb{E}\left[\sum_{n \geq 0} \mathbf{1}_{\left\{\zeta_{n}<2 \log \tilde{Y}\right\}} \mid \tilde{Y}\right]\right\},
\end{aligned}
$$

where $\tilde{Y}$ and $\left(\zeta_{n}\right)$ are independent. By (2.6),

$$
\mathbb{E}\left[\Sigma_{1}\right] \leq c_{17} \mathbb{E}\left[(\widetilde{Y})^{2} \int_{2 \log _{+} \tilde{Y}}^{\infty} \mathrm{e}^{-x} R(x) U(\mathrm{~d} x)+\tilde{Y} \int_{0}^{2 \log _{+} \tilde{Y}} R(x) U(\mathrm{~d} x)\right],
$$

which by (2.2) and (2.7) implies that

$$
\begin{aligned}
\mathbb{E}\left[\Sigma_{1}\right] & \leq c_{17} \mathbb{E}\left[(\tilde{Y})^{2} \int_{2 \log _{+} \tilde{Y}}^{\infty} \mathrm{e}^{-x}(x+1) U(\mathrm{~d} x)+\tilde{Y} \int_{0}^{2 \log _{+} \tilde{Y}}(x+1) U(\mathrm{~d} x)\right] \\
& \leq c_{18} \mathbb{E}\left[\tilde{Y}\left(1+\log _{+} \tilde{Y}\right)^{2}\right] \\
& =c_{18} \mathbb{E}\left[Y\left(1+\log _{+} Y\right)^{2}\right] .
\end{aligned}
$$


On the other hand, in the same way, we obtain

$$
\mathbb{E}\left[\Sigma_{2}\right] \leq c_{19} \mathbb{E}\left[Z\left(1+\log _{+} Z\right)\right] .
$$

Consequently,

$$
\mathbb{E}\left[\Sigma_{1}\right]+\mathbb{E}\left[\Sigma_{2}\right] \leq c_{20}\left(\mathbb{E}[Y+Z]+\mathbb{E}\left[Y\left(\log _{+} Y\right)^{2}\right]+\mathbb{E}\left[Z \log _{+} Z\right]\right) .
$$

Note that (3.1) ensures that $\mathbb{E}[Y+Z]<\infty$. Equation (3.7) is thus proved and we complete the proof of (3.4).

\subsection{Equation (1.3) is a necessary condition}

This section is devoted to proving that

$$
\max \left\{\mathbb{E}\left[Z \log _{+} Z\right], \mathbb{E}\left[Y\left(\log _{+} Y\right)^{2}\right]\right\}=\infty \quad \Longrightarrow \quad \mathbb{E}\left[D_{\infty}^{(0)}\right]=0 .
$$

Proof of (3.8). According to Theorem 3.1(ii), we need only to show that

$$
\sum_{n=1}^{\infty} \mathbb{E}_{\zeta_{n}}\left[X ; R\left(\zeta_{n}\right) \mathrm{e}^{-\zeta_{n}} X \geq y\right]=\infty, \quad \mathbb{P} \text {-a.s. for all } y>0 .
$$

We break the assumption on the left-hand side of (3.8) up into three cases. In each case, we find out a different lower bound for $X$ to establish (3.9). Hence, it follows that $D_{\infty}^{(0)}$ is trivial as $\mathbb{E}\left[D_{\infty}^{(0)}\right]=0$. The three cases are stated as follows:

$$
\begin{gathered}
\mathbb{E}\left[Y\left(\log _{+} Y\right)^{2}\right]=\infty, \quad \mathbb{E}\left[Y\left(\log _{+} Y\right)\right]<\infty, \\
\mathbb{E}\left[Y\left(\log _{+} Y\right)\right]=\infty, \\
\mathbb{E}\left[Z\left(\log _{+} Z\right)\right]=\infty .
\end{gathered}
$$

Proof of (3.9) under (3.10a). Recall that for any particle $x \in \mathbb{T} \backslash\{\varnothing\}, \Delta V(u)=V(u)-$ $V(\overleftarrow{u})$, and that under $\mathbb{P}_{a},(\Delta V(u) ;|u|=1)$ is distributed as $\mathcal{L}$. For any $s \in \mathbb{R}$, we define a pair of random variables

$$
Y_{+}(s):=\sum_{|u|=1} \mathrm{e}^{-\Delta V(u)} \mathbf{1}_{\{\Delta V(u)>-s\}}, \quad Y_{-}(s):=\sum_{|u|=1} \mathrm{e}^{-\Delta V(u)} \mathbf{1}_{\{\Delta V(u) \leq-s\}} .
$$

Clearly, $\widetilde{Y}=Y_{+}(s)+Y_{-}(s)$.

It follows from (3.6) and (2.2) that under $\mathbb{P}_{\zeta_{n}}$,

$$
\begin{aligned}
X & \geq \frac{\sum_{|u|=1} c_{1}\left(1+\zeta_{n}+\Delta V(u)\right) \mathrm{e}^{-\Delta V(u)} \mathbf{1}_{\left\{\Delta V(u)>-\zeta_{n} / 2\right\}}}{c_{2}\left(1+\zeta_{n}\right)} \\
& \geq \frac{\sum_{|u|=1} c_{1}\left(1 / 2+\zeta_{n} / 2\right) \mathrm{e}^{-\Delta V(u)} \mathbf{1}_{\left\{\Delta V(u)>-\zeta_{n} / 2\right\}}}{c_{2}\left(1+\zeta_{n}\right)} \\
& \geq c_{21} Y_{+}\left(\frac{\zeta_{n}}{2}\right),
\end{aligned}
$$

where $\left\{\left(Y_{+}(s), Y_{-}(s)\right) ; s \in \mathbb{R}\right\}$ is independent of $\zeta_{n}$ and $c_{21}:=c_{1} / 2 c_{2}>0$. Thus, it follows that, for any $y>0$,

$$
\sum_{n=1}^{\infty} \mathbb{E}\left[Y_{+}\left(\frac{\zeta_{n}}{2}\right) ; R\left(\frac{\zeta_{n}}{2}\right) \mathrm{e}^{-\zeta_{n}} Y_{+}\left(\frac{\zeta_{n}}{2}\right) \geq y \mid \zeta_{n}\right]=\infty, \quad \mathbb{P} \text {-a.s. }
$$


(3.9) holds. It is known that $\zeta_{n} \rightarrow \infty$ as $n$ goes to $\infty$ (see, e.g. [4]). It suffices that

$$
\sum_{n=1}^{\infty} F\left(\frac{\zeta_{n}}{2}, \zeta_{n}\right)=\infty, \quad \mathbb{P} \text {-a.s. }
$$

where $F(s, z):=\mathbb{E}\left[Y_{+}(s), \log Y_{+}(s) \geq z\right], s, z \in \mathbb{R}$.

Let $F_{1}(z):=\mathbb{E}[Y, \log Y \geq z]$, which is positive and nonincreasing. From Lemma 2.1 and (3.1), it follows that $\mathbb{E}[Y]=1$. Therefore, for any $s, z \in \mathbb{R}$,

$$
0 \leq F(s, z) \leq F_{1}(z) \leq \mathbb{E}[Y]=1 .
$$

On the one hand, from (3.10a), we obtain

$$
\begin{aligned}
\int_{0}^{\infty} F_{1}(y) y \mathrm{~d} y & =\int_{0}^{\infty} \mathbb{E}\left[Y \mathbf{1}_{\{\log Y \geq y\}}\right] y \mathrm{~d} y \\
& =\mathbb{E}\left[Y \int_{0}^{\left(\log _{+} Y\right)} y \mathrm{~d} y ; Y \geq 1\right] \\
& =\frac{\mathbb{E}\left[Y\left(\log _{+} Y\right)^{2}\right]}{2} \\
& =\infty .
\end{aligned}
$$

According to Proposition 2.1,

$$
\sum_{n=1}^{\infty} F_{1}\left(\zeta_{n}\right)=\infty, \quad \mathbb{P} \text {-a.s. }
$$

On the other hand, we can prove that $\sum_{n=1}^{\infty}\left[F_{1}\left(\zeta_{n}\right)-F\left(\zeta_{n} / 2, \zeta_{n}\right)\right]<\infty$, $\mathbb{P}$-a.s. In fact, as $Y=Y_{+}(s)+Y_{-}(s)$ under $\mathbb{P}$, for any $s, y \in \mathbb{R}$,

$$
\begin{aligned}
F_{1}(y)-F(s, y) & =\mathbb{E}\left[Y \mathbf{1}_{\{\log Y \geq y\}}-Y_{+}(s) \mathbf{1}_{\left\{\log Y_{+}(s) \geq y\right\}}\right] \\
& =\mathbb{E}\left[Y \mathbf{1}_{\left\{\log Y \geq y>\log Y_{+}(s)\right\}}+Y \mathbf{1}_{\left\{\log Y_{+}(s) \geq y\right\}}-Y_{+}(s) \mathbf{1}_{\left\{\log Y_{+}(s) \geq y\right\}}\right] \\
& =\mathbb{E}\left[Y \mathbf{1}_{\left\{\log Y \geq y>\log Y_{+}(s)\right\}}+Y_{-}(s) \mathbf{1}_{\left\{\log Y_{+}(s) \geq y\right\}}\right] .
\end{aligned}
$$

Note that $Y \leq 2 \max \left\{Y_{+}(s), Y_{-}(s)\right\}$ under $\mathbb{P}$. It follows that

$$
\begin{aligned}
F_{1}(y)-F(s, y) \leq & \mathbb{E}\left[2 Y_{-}(s) \mathbf{1}_{\left\{\log Y \geq y>\log Y_{+}(s), Y_{+}(s) \leq Y_{-}(s)\right\}}\right. \\
& \left.+Y \mathbf{1}_{\left\{\log Y \geq y>\log Y_{+}(s), Y_{+}(s)>Y_{-}(s)\right\}}\right]+\mathbb{E}\left[Y_{-}(s) \mathbf{1}_{\left\{\log Y_{-}(s) \geq y\right\}}\right] \\
\leq & 3 \mathbb{E}\left[Y_{-}(s)\right]+\mathbb{E}\left[Y \mathbf{1}_{\left\{\log Y \geq y>\log Y_{+}(s), Y_{+}(s)>Y_{-}(s)\right\}}\right] \\
\leq & 3 \mathbb{E}\left[Y_{-}(s)\right]+\mathbb{E}\left[Y \mathbf{1}_{\{\log Y \geq y>\log (Y / 2)\}}\right] \\
= & : d_{1}(s)+d_{2}(y) .
\end{aligned}
$$

As a consequence,

$$
\sum_{n=1}^{\infty}\left[F_{1}\left(\zeta_{n}\right)-f\left(\frac{\zeta_{n}}{2}, \zeta_{n}\right)\right] \leq \sum_{n \geq 0} d_{1}\left(\frac{\zeta_{n}}{2}\right)+\sum_{n \geq 0} d_{2}\left(\zeta_{n}\right)
$$


Taking the expectation on both sides yields

$$
\begin{aligned}
\mathbb{E}\left[\sum_{n=1}^{\infty}\left(F_{1}\left(\zeta_{n}\right)-f\left(\frac{\zeta_{n}}{2}, \zeta_{n}\right)\right)\right] & \leq \mathbb{E}\left[\sum_{n \geq 0} d_{1}\left(\frac{\zeta_{n}}{2}\right)\right]+\mathbb{E}\left[\sum_{n \geq 0} d_{2}\left(\zeta_{n}\right)\right] \\
& =\int_{0}^{\infty} d_{1}\left(\frac{x}{2}\right) R(x) U(\mathrm{~d} x)+\int_{0}^{\infty} d_{2}(x) R(x) U(\mathrm{~d} x),
\end{aligned}
$$

where the last equality comes from (2.6).

For the first integration, from Lemma 2.1, we obtain

$$
d_{1}(s)=3 \mathbb{E}\left[Y_{-}(s)\right]=3 \mathbb{E}\left[\sum_{|x|=1} \mathrm{e}^{-V(x)} \mathbf{1}_{\{V(x) \leq-s\}}\right]=3 \mathbb{P}\left(-S_{1} \geq s\right) .
$$

By (2.2), (2.7), and (3.1), we have

$$
\begin{aligned}
\int_{0}^{\infty} d_{1}\left(\frac{x}{2}\right) R(x) U(\mathrm{~d} x) & =3 \int_{0}^{\infty} \mathbb{P}\left(-2 S_{1} \geq x\right) R(x) U(\mathrm{~d} x) \\
& =3 \mathbb{E}\left[\int_{0}^{-2 S_{1}} R(x) U(\mathrm{~d} x) ;-2 S_{1} \geq 0\right] \\
& \leq c_{22} \mathbb{E}\left[\left(1+\left(-2 S_{1}\right)_{+}\right)^{2}\right] \\
& <\infty .
\end{aligned}
$$

For the second integration on the right-hand side of (3.13), as $d_{2}(y)=\mathbb{E}\left[Y \mathbf{1}_{\{\log Y \geq y>\log (Y / 2)\}}\right]$, we use (2.2), (2.7), and (3.10a) to obtain

$$
\begin{aligned}
\int_{0}^{\infty} d_{2}(x) R(x) U(\mathrm{~d} x) & =\int_{0}^{\infty} \mathbb{E}\left[Y \mathbf{1}_{\{\log Y \geq x>\log (Y / 2)\}}\right] R(x) U(\mathrm{~d} x) \\
& =\mathbb{E}\left[Y \int_{(\log Y-\log 2)_{+}}^{\log _{+} Y} R(x) U(\mathrm{~d} x)\right] \\
& \leq c_{23} \mathbb{E}\left[Y\left(1+\log _{+} Y\right)\right] \\
& <\infty
\end{aligned}
$$

Returning to (3.13), we conclude that

$$
\mathbb{E}\left[\sum_{n=1}^{\infty}\left(F_{1}\left(\zeta_{n}\right)-f\left(\frac{\zeta_{n}}{2}, \zeta_{n}\right)\right)\right] \leq \mathbb{E}\left[\sum_{n \geq 0} d_{1}\left(\frac{\zeta_{n}}{2}\right)\right]+\mathbb{E}\left[\sum_{n \geq 0} d_{2}\left(\zeta_{n}\right)\right]<\infty
$$

Therefore,

$$
\sum_{n=1}^{\infty}\left[F_{a}\left(\zeta_{n}\right)-f\left(\frac{\zeta_{n}}{2}, \zeta_{n}\right)\right]<\infty, \quad \mathbb{P} \text {-a.s. }
$$

which, combined with (3.12), implies (3.11). Thus, (3.9) is proved under (3.10a).

Proof of (3.9) under (3.10b). Now we suppose that $\mathbb{E}\left[Y \log _{+} Y\right]=\infty$. By (2.2), we observe that under $\mathbb{P}_{\zeta_{n}}$,

$$
X=\frac{\sum_{|u|=1} R\left(\Delta V(u)+\zeta_{n}\right) \mathrm{e}^{-\Delta V(u)} \mathbf{1}_{\left\{\Delta V(u)>-\zeta_{n}\right\}}}{R\left(\zeta_{n}\right)} \geq c_{1} \frac{Y_{+}\left(\zeta_{n}\right)}{R\left(\zeta_{n}\right)},
$$

where $\left\{Y_{+}(s) ; s \in \mathbb{R}\right\}$ and $\zeta_{n}$ are independent. 
To establish (3.9), we need only to show that, for any $y \geq 1$,

$$
\sum_{n \geq 1} \mathbb{E}\left[\frac{Y_{+}\left(\zeta_{n}\right)}{R\left(\zeta_{n}\right)} ; Y_{+}\left(\zeta_{n}\right) \geq y \mathrm{e}^{\zeta_{n}} \mid \zeta_{n}\right]=\sum_{n \geq 1} \frac{F\left(\zeta_{n}, \log y+\zeta_{n}\right)}{R\left(\zeta_{n}\right)}=\infty, \quad \mathbb{P} \text {-a.s. }
$$

For any $y \geq 1$ fixed, let

$$
F_{2}(x):=\frac{F_{1}(\log y+x)}{R(x)} \text { for all } x \geq 0,
$$

which is nonincreasing as $R(x)=U^{-}([0, x))$ is nondecreasing and $F_{1}$ is nonincreasing. We have

$$
\sum_{n \geq 1} F_{2}\left(\zeta_{n}\right)=\sum_{n \geq 1} \frac{F\left(\zeta_{n}, \log y+\zeta_{n}\right)}{R\left(\zeta_{n}\right)}+\sum_{n \geq 1} \frac{F_{1}\left(\log y+\zeta_{n}\right)-F\left(\zeta_{n}, \log y+\zeta_{n}\right)}{R\left(\zeta_{n}\right)}
$$

By (2.2),

$$
\frac{F_{1}(\log y+x)}{c_{2}(1+x)} \leq F_{2}(x) \leq \frac{1}{c_{1}} .
$$

From (3.10b), it follows that

$$
\begin{aligned}
\int_{0}^{\infty} F_{2}(x) x \mathrm{~d} x & \geq \int_{0}^{\infty} F_{1}(\log y+x) \frac{x}{c_{2}(1+x)} \mathrm{d} x \\
& \geq \int_{1}^{\infty} c_{24} \mathbb{E}\left[Y \mathbf{1}_{\{\log Y \geq \log y+x\}}\right] \mathrm{d} x \\
& \geq c_{24} \mathbb{E}\left[Y(\log Y-\log y-1)_{+}\right] \\
& =\infty
\end{aligned}
$$

By Proposition 2.1,

$$
\sum_{n \geq 0} F_{2}\left(\zeta_{n}\right)=\sum_{n \geq 0} \frac{F_{1}\left(\log y+\zeta_{n}\right)}{R\left(\zeta_{n}\right)}=\infty, \quad \mathbb{P} \text {-a.s. }
$$

In view of (3.14) and (3.15), it suffices to show that

$$
\sum_{n \geq 0} \frac{F_{1}\left(\log y+\zeta_{n}\right)-F\left(\zeta_{n}, \log y+\zeta_{n}\right)}{R\left(\zeta_{n}\right)}<\infty, \quad \mathbb{P} \text {-a.s. }
$$

Recall that $F_{1}(z)-F(s, z) \leq d_{1}(s)+d_{2}(z)$. By (2.6),

$$
\begin{aligned}
\mathbb{E}\left[\sum_{n \geq 0} \frac{F_{1}\left(\log y+\zeta_{n}\right)-F\left(\zeta_{n}, \log y+\zeta_{n}\right)}{R\left(\zeta_{n}\right)}\right] & \leq \mathbb{E}\left[\sum_{n \geq 0} \frac{d_{1}\left(\zeta_{n}\right)+d_{2}\left(\log y+\zeta_{n}\right)}{R\left(\zeta_{n}\right)}\right] \\
& =\int_{0}^{\infty}\left[d_{1}(x)+d_{2}(\log y+x)\right] U(\mathrm{~d} x)
\end{aligned}
$$


On the one hand, recalling that $d_{1}(x)=3 \mathbb{P}\left(-S_{1} \geq x\right)$, from (2.7), we obtain

$$
\begin{aligned}
\int_{0}^{\infty} d_{1}(x) U(\mathrm{~d} x) & =\int_{0}^{\infty} 3 \mathbb{P}\left(-S_{1} \geq x\right) U(\mathrm{~d} x) \\
& =3 \mathbb{E}\left[\int_{0}^{\left(-S_{1}\right)_{+}} U(\mathrm{~d} x)\right] \\
& \leq 3 c_{4} \mathbb{E}\left[1+\left(-S_{1}\right)_{+}\right] \\
& <\infty
\end{aligned}
$$

On the other hand, recalling that $d_{2}(x)=\mathbb{E}[Y ; \log Y \geq x>\log Y-\log 2]$, by (2.7) again, we obtain

$$
\begin{aligned}
\int_{0}^{\infty} d_{2}(\log y+x) U(\mathrm{~d} x) & =\int_{0}^{\infty} \mathbb{E}\left[Y \mathbf{1}_{\{\log Y \geq \log y+x>\log Y-\log 2\}}\right] U(\mathrm{~d} x) \\
& =\mathbb{E}\left[Y \int_{(\log Y-\log y-\log 2)_{+}}^{(\log Y-\log y)_{+}} U(\mathrm{~d} x)\right] \\
& \leq c_{4}(1+\log 2) \mathbb{E}[Y] \\
& <\infty
\end{aligned}
$$

Combined with (3.18) and (3.19), (3.17) becomes

$$
\mathbb{E}\left[\sum_{n \geq 1} \frac{F_{1}\left(\log y+\zeta_{n}\right)-F\left(\zeta_{n}, \log y+\zeta_{n}\right)}{R\left(\zeta_{n}\right)}\right]<\infty .
$$

Thus, we obtain (3.16), which completes the proof of (3.9) given (3.10b).

Proof of (3.9) under (3.10c). In this part we assume that $\mathbb{E}\left[Z \log _{+} Z\right]=\infty$ with $Z=$ $\sum_{|u|=1} V(u)_{+} \mathrm{e}^{-V(u)} \geq 0$. We observe that under $\mathbb{P}_{\zeta_{n}}$,

$$
X \geq \frac{\sum_{|u|=1} R\left(\Delta V(u)+\zeta_{n}\right) \mathrm{e}^{-\Delta V(u)} \mathbf{1}_{\{\Delta V(u)>0\}}}{R\left(\zeta_{n}\right)} \geq \frac{c_{1}}{R\left(\zeta_{n}\right)} \widetilde{Z},
$$

where $\widetilde{Z}=\sum_{|x|=1}(\Delta V(x))_{+} \mathrm{e}^{-\Delta V(x)}$ is independent of $\zeta_{n}$. As a consequence, for any $y>0$,

$$
\sum_{n \geq 1} \mathbb{E}_{\zeta_{n}}\left[X ; R\left(\zeta_{n}\right) \mathrm{e}^{-\zeta_{n}} X \geq y\right] \geq \sum_{n \geq 1} \frac{c_{1}}{R\left(\zeta_{n}\right)} \mathbb{E}\left[\widetilde{Z} ; c_{1} \widetilde{Z} \geq y \mathrm{e}^{\zeta_{n}} \mid \zeta_{n}\right]
$$

Recall that $\widetilde{Z}$ is distributed as $Z$ under $\mathbb{P}$. Therefore, it is sufficient to prove that, for any $y>0$,

$$
\sum_{n \geq 1} \frac{1}{R\left(\zeta_{n}\right)} \mathbb{E}\left[\tilde{Z} ; \tilde{Z} \geq y \mathrm{e}^{\zeta_{n}} \mid \zeta_{n}\right]=\sum_{n \geq 1} F_{3}\left(\zeta_{n}\right)=\infty, \quad \mathbb{P} \text {-a.s. }
$$

where

$$
F_{3}(z):=\frac{\mathbb{E}[Z ; \log Z \geq z+\log y]}{R(z)} \text { for all } z \geq 0 .
$$

Since $R$ is nondecreasing, the function $F_{3}$ is nonincreasing. By Lemma 2.1 and (2.2),

$$
0 \leq F_{3}(z) \leq \frac{\mathbb{E}[Z]}{R(z)} \leq \frac{\mathbb{E}\left[\left(S_{1}\right)_{+}\right]}{c_{1}}<\infty
$$


Moreover, by (2.7) and (3.10c),

$$
\begin{aligned}
\int_{0}^{\infty} F_{3}(x) x \mathrm{~d} x & \geq \int_{1}^{\infty} c_{25} \mathbb{E}[Z ; \log Z-\log y \geq x] \mathrm{d} x \\
& \geq c_{25} \mathbb{E}\left[Z(\log Z-\log y-1)_{+}\right] \\
& =\infty
\end{aligned}
$$

Due to Proposition 2.1, for any $y>0$, we obtain

$$
\sum_{n \geq 1} \frac{1}{R\left(\zeta_{n}\right)} \mathbb{E}\left[\tilde{Z} ; \tilde{Z} \geq y \mathrm{e}^{\zeta_{n}} \mid \zeta_{n}\right]=\sum_{n \geq 1} F_{3}\left(\zeta_{n}\right)=\infty, \quad \mathbb{P} \text {-a.s. }
$$

which completes the proof of (3.9) under (3.10c).

\section{Acknowledgements}

I would like to thank Professor John Biggins and Professor Zhan Shi for valuable discussions. I am also grateful to the referee for his/her careful reading and helpful comments.

\section{References}

[1] AïрÉKon, E. (2013). Convergence in law of the minimum of a branching random walk. Ann. Prob. 41, 13621426.

[2] Bertoin, J. and Doney, R. A. (1994). On conditioning a random walk to stay nonnegative. Ann. Prob. 22, 2152-2167.

[3] Biggins, J. D. (1977). Chernoff's theorem in the branching random walk. J. Appl. Prob. 14, 630-636.

[4] Biggins, J. D. (2003). Random walk conditioned to stay positive. J. London Math. Soc. (2) 67, $259-272$.

[5] Biggins, J. D. And Kyprianou, A. E. (1997). Seneta-Heyde norming in the branching random walk. Ann. Prob. 25, 337-360.

[6] Biggins, J. D. And Kyprianou, A. E. (2004). Measure change in multitype branching. Adv. Appl. Prob. 36, 544-581.

[7] Biggins, J. D. And Kyprianou, A. E. (2005). Fixed points of the smoothing transform: the boundary case. Electron. J. Prob. 10, 609-631.

[8] Chauvin, B. And Rouault, A. (1988). KPP equation and supercritical branching Brownian motion in the subcritical speed area. Application to spatial trees. Prob. Theory Relat. Fields 80, 299-314.

[9] Feller, W. (1971). An Introduction to Probability Theory and Its Applications, Vol. II, 2nd edn. John Wiley, New York.

[10] Harris, S. C. And Roberts, M. I. (2014). The many-to-few lemma and multiple spines. Preprint. Available at http://arxiv.org/abs/1106.4761.

[11] Hu, Y. AND SHI, Z. (2009). Minimal position and critical martingale convergence in branching random walks, and directed polymers on disordered trees. Ann. Prob. 37, 742-789.

[12] LIU, Q. (1998). Fixed points of a generalized smoothing transformation and applications to the branching random walk. Adv. Appl. Prob. 30, 85-112.

[13] Liu, Q. (2000). On generalized multiplicative cascades. Stoch. Process Appl. 86, 263-286.

[14] Lyons, R. (1997). A simple path to Biggins' martingale convergence for branching random walk. In Classical and Modern Branching Processes (IMA Vol. Math. Appl. 84), Springer, New York, pp. 217-221.

[15] Revuz, D. And Yor, M. (1999). Continuous Martingales and Brownian Motion, 3rd edn. Springer, Berlin.

[16] TANAKA, H. (1989). Time reversal of random walks in one-dimension. Tokyo J. Math. 12, 159-174.

[17] Vatutin, V. A. And Wachtel, V. (2009). Local probabilities for random walks conditioned to stay positive. Prob. Theory Relat. Fields 143, 177-217.

[18] YANG, T. AND REN, Y.-X. (2011). Limit theorem for derivative martingale at criticality w.r.t. branching Brownian motion. Statist. Prob. Lett. 81, 195-200. 